\title{
Mammalian target of rapamycin signaling activation patterns in neuroendocrine tumors of the lung
}

\author{
Luisella Righi, Marco Volante, Ida Rapa, Veronica Tavaglione, \\ Frediano Inzani ${ }^{1}$, Giuseppe Pelosi ${ }^{2}$ and Mauro Papotti
}

\author{
Division of Pathology, Department of Clinical and Biological Sciences, University of Turin at San Luigi Hospital, \\ Regione Gonzole 10, 10043 Orbassano, Torino, Italy \\ ${ }^{1}$ Division of Pathology, University of Parma, 43100 Parma, Italy \\ ${ }^{2}$ National Cancer Institute and University of Milan, 20133 Milan, Italy \\ (Correspondence should be addressed to L Righi; Email: luisella.righi@unito.it)
}

\begin{abstract}
Among alternative therapeutic strategies in clinically aggressive neuroendocrine tumors (NETs) of the lung, promising results have been obtained in experimental clinical trials with mammalian target of rapamycin (mTOR) inhibitors, though in the absence of a proven mTOR signaling activation status. This study analyzed the expression of phosphorylated mTOR (p-mTOR) and its major targets, the ribosomal p70S6-kinase (S6K) and the eukaryotic initiation factor 4E-binding protein 1 (4EBP1) in a large series of 218 surgically resected, malignant lung NETs, including 24 metastasizing typical carcinoids, 73 atypical carcinoids, 60 large cell neuroendocrine carcinomas (LCNECs), and 61 small cell carcinomas (SCLCs). By immunohistochemistry, lowto-intermediate-grade tumors as compared with high-grade tumors showed higher levels of p-mTOR and phosphorylated S6K (p-S6K) $(P<0.001)$, at variance with phosphorylated 4EBP1 ( $p$-4EBP1), which was mainly expressed in LCNECs and SCLCs $(P<0.001)$. The activated status of mTOR pathway was proved by the strong correlation of $p$-mTOR with $p$-S6K and somatostatin receptor(s). Western blot analysis of NET tumor samples confirmed such findings, and differential sensitivity to mTOR inhibition according to mTOR pathway activation characteristics was determined in two lung carcinoid cell lines in vitro. None of the investigated molecules had an impact on survival. However, in low-grade tumors, low p-mTOR expression correlated with lymph node metastases $(P=0.016)$, recurrent disease, and survival $(P=0.005)$. In conclusion, these data demonstrate a differential mTOR activation status in the spectrum of pulmonary NETs, possibly suggesting that $\mathrm{mTOR}$ pathway profiling might play a predictive role in candidate patients for mTOR-targeted therapies.
\end{abstract}

Endocrine-Related Cancer (2010) 17 977-987

\section{Introduction}

The management of lung neuroendocrine tumors (NETs) mainly depends on both grade of differentiation (low-to-intermediate versus high-grade (HG)) and clinical stage at diagnosis (localized versus metastatic). Surgery is the treatment of choice for low-to-intermediate grade (i.e. typical carcinoids (TCs) or atypical carcinoids (ACs)) and localized tumors, while in $\mathrm{HG}$ and/or disseminated lesions chemotherapy is generally preferred (Pelosi et al. 2006, Garcia-Yuste et al. 2008). Traditional therapies offer limited benefits to patients with advanced disease: the traditional
DNA-damaging cytotoxic agents (i.e. platinum-based drugs) have low efficacy and although a large number of therapeutic options have been explored, there is little consensus on a single standard treatment approach (Srirajaskanthan et al. 2009), especially in the group of clinically aggressive bronchial carcinoids.

Emerging data on the molecular mechanisms of carcinogenesis and tumor progression prompted a new era of molecular therapeutics with the development of selective targeted agents. In this context, there are several, yet poorly explored, potential therapeutic options for lung NETs including somatostatin analogs, 
inhibitors of the vascular endothelial growth factor (VEGF) pathway, and inhibitors of the mammalian target of rapamycin (mTOR), which have shown promising activity in recent clinical studies (Duran et al. 2007, Kulke 2007, Yao et al. 2008, 2010).

mTOR is a serine threonine kinase, which participates in the regulation of proliferation, cell growth, and apoptosis through modulation of cell cycle progression (Vignot et al. 2005). The activated phosphorylated mTOR (p-mTOR) kinase leads to the subsequent phosphorylation of downstream effectors: the ribosomal p70S6-kinase (S6K) and the eukaryotic initiation factor 4E-binding protein 1 (4EBP1), two key proteins that regulate translation of mRNAs into proteins required for cell cycle progression from $\mathrm{G}_{1}$ to $\mathrm{S}$ phase (Podsypanina et al. 2001, Dancey 2006). Recent insights revealed a significant complexity of the mTOR pathway that seems to cross talk with other well-characterized signaling cascades, thus paving the way for the use of combined therapies (Bjornsti \& Houghton 2004, Guertin \& Sabatini 2007, Meric-Bernstam \& Gonzalez-Angulo 2009). mTOR signaling pathway can be upstream activated - most commonly via the PI3 kinase (PI3K)/AKT pathway by receptors such as somatostatin receptors (SSTRs) or insulin-like growth factor receptor 1 (IGF1R) or by loss of inhibiting molecules, such as PTEN (von Wichert et al. 2000, Wang et al. 2002). Rapamycin (Sirolimus, Wyeth, Philadelphia, PA, USA) and its derivates are immunosuppressive macrolides that specifically block mTOR signaling and have been shown to possess anti-proliferative activity in a variety of malignancies both in vitro (Zitzmann et al. 2007, Grozinsky-Glasberg et al. 2010, Missiaglia et al. 2010) and in phase II clinical trials (Yao et al. 2008). Inhibition of mTOR prevents phosphorylation of S6K, 4EBP1, and, indirectly, other proteins involved in the transcription and cell cycle control, leading to $G_{1}$ phase cell growth arrest.

Two rapamycin derivates have recently been evaluated in patients with NETs: temsirolimus (CCI779; Wyeth, Madison, NJ, USA) and everolimus (RAD001; Novartis Pharma AG). A multicentric study has recently demonstrated that temsirolimus effectively down-regulates the phosphorylation of S6K and that higher baseline levels of phosphorylated S6K (p-S6K) and p-mTOR seem to predict a better response in advanced neuroendocrine (NE) carcinomas (Duran et al. 2006), although temsirolimus does not modify the progression-free survival in advanced small cell lung cancer patients (Pandya et al. 2007). New perspectives flow from phase I trials aimed to determine the safety, tolerability, pharmacokinetics, and pharmacodynamics of novel mTOR inhibitors such as deforolimus (AP23573; Ariad Pharmaceuticals, Cambridge, MA, USA), which was well tolerated and showed encouraging antitumor activity (Mita et al. 2008). Furthermore, in vitro studies and in vivo clinical trials combining mTOR inhibitors and the somatostatin analog octreotide have recently been published with controversial results in terms of additive anti-tumoral effects of the two compounds (Grozinsky-Glasberg et al. 2008, Moreno et al. 2008, Yao et al. 2008).

Despite all the above pre-clinical and clinical studies on the anti-neoplastic efficacy of mTOR inhibitors in a variety of tumors, data on the activation status of mTOR signaling cascade in pulmonary NET are still lacking. In this respect, a detailed protein expression map of mTOR-pathway-related molecules in lung NET could not only define specific expression patterns predictive of clinical response, as suggested for other malignancies (Lam et al. 2007), but also investigate the prognostic implications of these molecules. Therefore, aim of this study is to evaluate the expression of activated mTOR-related proteins in a large series of pulmonary NET - with special reference to clinically malignant cases.

\section{Materials and methods}

\section{Case selection}

Eight hundred and eighty-three surgically resected NETs of the lung were recorded between 1989 and 2007 in the pathology files of the Universities of Turin and Parma and the European Institute of Oncology of Milan (467, 188, and 217 cases respectively). Among them, a series of 218 clinically malignant lesions (129 cases from Turin, 40 from Parma, and 49 from Milan) was collected, including 24 TCs with lymph node metastases at the time of the diagnosis (TC mets), 73 ACs with or without lymph node metastases, 60 large cell neuroendocrine carcinomas (LCNECs), and 61 small cell carcinomas (SCLCs). Pathological samples corresponded to primaries in all but 15 cases, where lymph node metastases were the only available material. In 15 cases, primary tumor and the corresponding lymph node metastasis were analyzed. All cases were classified according to the last 2004 WHO classification on lung tumors (Travis et al. 2004) and the clinical and pathological characteristics were reported in detail elsewhere (Righi et al. 2010) and are summarized in Table 1. Forty consecutive non metastatic TCs were collected from the files of the University of Turin to be used as control group for the baseline expression of the markers under evaluation. All cases were anonymized by a pathology staff 
Table 1 Summary of clinico-pathological features of 218 aggressive pulmonary neuroendocrine tumors (see also Righi et al. 2010)

\begin{tabular}{lllll}
\hline & $\begin{array}{l}\text { TC mets } \\
(\# 24)\end{array}$ & $\begin{array}{l}\text { AC } \\
(\# 73)\end{array}$ & $\begin{array}{l}\text { LCNEC } \\
(\# 60)\end{array}$ & $\begin{array}{l}\text { SCLC } \\
(\# 61)\end{array}$ \\
\hline M/F ratio & $1 / 1$ & $1.3 / 1$ & $7.5 / 1$ & $4 / 1$ \\
Age, mean (years) & 48 & 55 & 64 & 65 \\
Tumor size, mean (cm) & 25 & 32 & 42 & 38 \\
Positive nodal status (\%) & 100 & 47 & 39 & 61 \\
Advanced stage & 35 & 23 & 28 & 37 \\
$\quad$ (IIIA-IIIB-IV) (\%) & & & & \\
AWD/DOD status (\%) & 8 & 35 & 65 & 65 \\
Ki-67 (mean \%) & 3 & 16 & 70 & 76 \\
SSTR2A expression & 71 & 51 & 33 & 38 \\
SSTR3 expression & 58 & 45 & 33 & 29 \\
\hline
\end{tabular}

TC mets, typical carcinoid with metastases; AC, atypical carcinoid; LCNEC, large cell neuroendocrine carcinoma; SCLC, small cell lung carcinoma; $M$, male; $F$, female; AWD, alive with disease; DOD, dead of disease; SSTR, somatostatin receptor.

member not involved in the study. Clinical data were compared and analyzed through coded data only. The study was approved by the institutional review board of the hospital.

\section{Immunohistochemistry}

Immunohistochemistry was performed using the primary monoclonal antibodies listed in Table 2; all antibodies were purchased from Cell Signaling Technologies (Beverly, MA, USA). Five micron-thick paraffin sections were collected on the charged slides, deparaffinized, and re-hydrated in water. After antigen retrieval in $\mathrm{pH} 6.0$ citrate buffer for $5 \mathrm{~min}$ at $125^{\circ} \mathrm{C}$ in a pressure cooker, the relevant primary antibodies were incubated overnight at $4{ }^{\circ} \mathrm{C}$. Immunoreactions were revealed by a biotin-free dextran-chain detection system (Envision, DakoCytomation, Glostrup, Denmark) and were developed using $3^{\prime}, 3^{\prime}$-diaminobenzidine as the chromogen. The specificity of all reactions was validated in parallel control sections omitting the primary antibodies for each immunohistochemical run.

\section{Immunohistochemical data interpretation}

Immunohistochemical findings were evaluated independently by two of us ( $\mathrm{L} \mathrm{R}$ and $\mathrm{M} \mathrm{V}$ ) and cases with conflicting scores were reviewed jointly by a multi-head microscope until a consensus was reached. All cases were evaluated using a semi-quantitative histological score ( $H$-score) (Huang et al. 2005, Cappia et al. 2008) taking into account both the percentage of positive tumor population within the whole section and the immunostaining intensity evaluated subjectively as being negative (0), weak (1), moderate (2), and strong (3). For each case, the $H$-score was obtained by multiplying the percentages of reactive cells by the corresponding immunostaining intensity, thereby obtaining a final score ranging from 0 to 300 .

\section{Western blot analysis}

Thirteen frozen lung NET samples, not included in the present series of 218 cases, were available from the tissue bank of the Pathology Unit at the University of Turin. All samples were homogenized and lysated in TNE lysis buffer supplemented with $1 \%$ protease inhibitor cocktail (Complete, Roche Diagnostic Corporation). The protein concentration was evaluated using the BCA protein assay kit (Pierce, Milwaukee, WI, USA), and $50 \mu \mathrm{g}$ protein were resolved in $8 \%$ SDS-PAGE and transferred to nitrocellulose membranes for each experiment. The membrane blots were blocked for $1 \mathrm{~h}$ with 5\% BSA in TBS-Tween $0.1 \%$ and incubated overnight at $4{ }^{\circ} \mathrm{C}$ with the primary antibodies listed in Table 2 (all from Cell Signaling Technology except for $\beta$-actin from Santa Cruz Biotechnology, Santa Cruz, CA, USA). Immunoreactive proteins were visualized using HRP-conjugated anti-mouse or anti-rabbit antibody (1:3000 and 1:1000 respectively) and enhanced chemioluminescence (Amersham Biosciences) as a substrate. All western blots experiments were repeated twice and showed consistent results. The same cases were also tested for comparison by means of immunohistochemistry for p-mTOR, p-S6K, and phosphorylated 4EBP1 (p-4EBP1).

Table 2 List of primary antibodies used for western blot and immunohistochemistry

\begin{tabular}{llllll}
\hline Primary antibody & Clone & Specificity & Dilution & Use & Source \\
\hline Phospho-mTOR & Rabbit, 49F9 & Ser2448 & $1 / 100$ IHC 1/1000 WB & WB, IHC & Cell Signaling \\
Phospho-p70S6K & Mouse, 1A5 & Thr389 & $1 / 400$ IHC 1/1000 WB & WB, IHC & Cell Signaling \\
Phospho-4EBP1 & Rabbit, 236B4 & Thr37/46 & $1 / 300$ IHC 1/1000 WB & WB, IHC & Cell Signaling \\
4EBP1 & Rabbit, polyclonal & / & $1 / 1000$ & WB & Cell Signaling \\
Phospho-AKT & Rabbit, 736E11 & Ser473 & $1 / 1000$ & WB & Cell Signaling \\
$\beta-A c t i n$ & Mouse, C4 & $/$ & $1 / 1000$ & WB & Santa Cruz
\end{tabular}

IHC, immunohistochemistry; WB, western blot. 


\section{Cell culture and proliferation assay}

Two human lung cell lines, TC (H727) and AC (H720), were purchased from ATCC (LGC Standards s.r.l., Sesto San Giovanni, Milan, Italy). Cell lines were maintained in RPMI medium (H727) and in $1: 1$ DMEM/F12 mixture (H720) supplemented with $10 \%$ FCS, $2 \mathrm{nM}$ glutamine, penicillin $(25 \mathrm{U} / \mathrm{ml})$, and streptomycin $(25 \mu \mathrm{g} / \mathrm{ml}$, all from Sigma-Aldrich) in a humidified atmosphere containing $5 \% \mathrm{CO}_{2}$ at $37^{\circ} \mathrm{C}$. The cell lines were treated with rapamycin at different concentrations $(0.1,1$, and $10 \mathrm{nmol} / \mathrm{l}$; Calbiochem, Darmstadt, Germany) or with RAD001 (0.1, 1, and $10 \mathrm{nmol} / \mathrm{l}$; Novartis Pharma AG). Cells were incubated with $0.1 \%$ dimethyl sulfoxide (Sigma-Aldrich) served as control. Cells were plated onto 96 multiwell plates in triplicate. After overnight incubation at $37^{\circ} \mathrm{C}$, cells were treated at increasing doses of rapamycin or RAD001 for 48 and $72 \mathrm{~h}$. Cell viability was evaluated by adding $0.5 \mathrm{mg} / \mathrm{ml}$ methyl thiazolyl tetrazolium (MTT; Sigma-Aldrich) solution, incubating for $4 \mathrm{~h}$, and then adding $100 \mu \mathrm{m}$ dimethyl sulfoxide. The $570 \mathrm{~nm}$ absorbance was measured using a microplate reader (Model 540, Bio-Rad). Western blot experiments on protein extracts at basal or after $24 \mathrm{~h}$ of treatment were performed as described above in duplicate.

\section{Statistical analysis}

Statistical analysis was performed using Graphpad 4 software (Graphpad Software, La Jolla, CA, USA) and the results were considered statistically significant at a level of $P<0.05$. One-way ANOVA and non parametric Mann-Whitney $U$ tests were used to compare the distribution of the markers investigated among the different tumor groups and with respect to the clinical pathological variables. The Spearman's test was used to analyze the correlation index among markers expression. Overall, survival analysis was performed using the Kaplan-Meier method and Log-Rank test.

\section{Results}

\section{Distribution of mTOR signaling molecules in lung NETs}

Immunohistochemical staining for p-mTOR and p-4EBP1 provided the expected cytoplasmic pattern, while p-S6K showed either a cytoplasmic perinuclear dot-like or a diffuse nuclear pattern of staining.

In peri-tumoral non-neoplastic parenchyma, a weak p-mTOR, p-S6K, and p-4EBP1 immunoreactivity were observed in normal bronchial epithelium and endothelia. Alveolar histiocytes were also reactive for $\mathrm{p}-\mathrm{S} 6 \mathrm{~K}$ and $\mathrm{p}-4 \mathrm{EBP} 1$, whereas a strong $\mathrm{p}-\mathrm{mTOR}$ immunoreactivity was detected in reactive alveolar epithelial cells at the periphery of the tumors (data not shown).

Distribution of p-mTOR and its downstream activation molecules was significantly different among the various NET types (all $P<0.0001$; Fig. 1). In particular, p-mTOR and p-S6K were expressed at higher levels in low-to-intermediategrade tumors (low-grade (LGs), corresponding to TC mets and ACs) as compared with those in $\mathrm{HG}$ carcinomas (HGs, corresponding to LCNECs and SCLCs $)(P<0.001$ and $P=0.027$ respectively), whereas an opposite distribution held true for p-4EBP1 in LG and $\mathrm{HG}$ tumors respectively $(P<0.001)$. In the group of LG tumors, TC mets showed the highest mean $H$-score values for p-mTOR and p-4EBP1, albeit statistically not different as compared with the control TC and AC groups. At variance, p-S6K $H$-score distribution was similar in control TC and TC mets, but significantly lower in AC as compared with both TC mets and control TC $(P<0.001)$. Notably, a wide dispersion of $H$-score values was detected within individual tumor groups (range of $\mathrm{H}$-score being 0-220, 0-220, 0-170, and 0-110 for TC mets, AC, LCNEC, and SCLC respectively). In the 15 cases where metastatic tumor tissue was compared with the primary lesion, no significant differences were observed in terms of both the intensity and the percentage of positive cells for any of the three markers under investigation.

Western blot analysis (Fig. 2) confirmed the heterogeneity of mTOR pathway activation in lung NETs. P-mTOR and p-S6K and, more markedly, p-AKT proteins were expressed consistently in carcinoid samples, both TC and AC. By contrast, in HG tumor samples, both p-mTOR and p-S6K were negative or weakly positive, except for one case with p-S6K. In parallel, p-AKT expression was preserved, though to a generally lower extent, thus suggesting the activation of alternative AKT-mediated signaling pathways in these tumors. Total 4EBP1 was detectable in all samples with a comparable intensity, whereas p-4EBP1 was more heterogeneously present, with a higher intensity in LCNEC cases, in line with our observation that LCNEC had its highest expression by means of immunohistochemistry. Parallel immunohistochemical determination of p-mTOR and p-S6K, evaluated in all cases, was consistent with the western blot results. Conversely, p-4EBP1 showed a slightly lower direct concordance mainly due to the presence of high immunohistochemical $H$-score in cases with weak western blot bands, with special reference to 

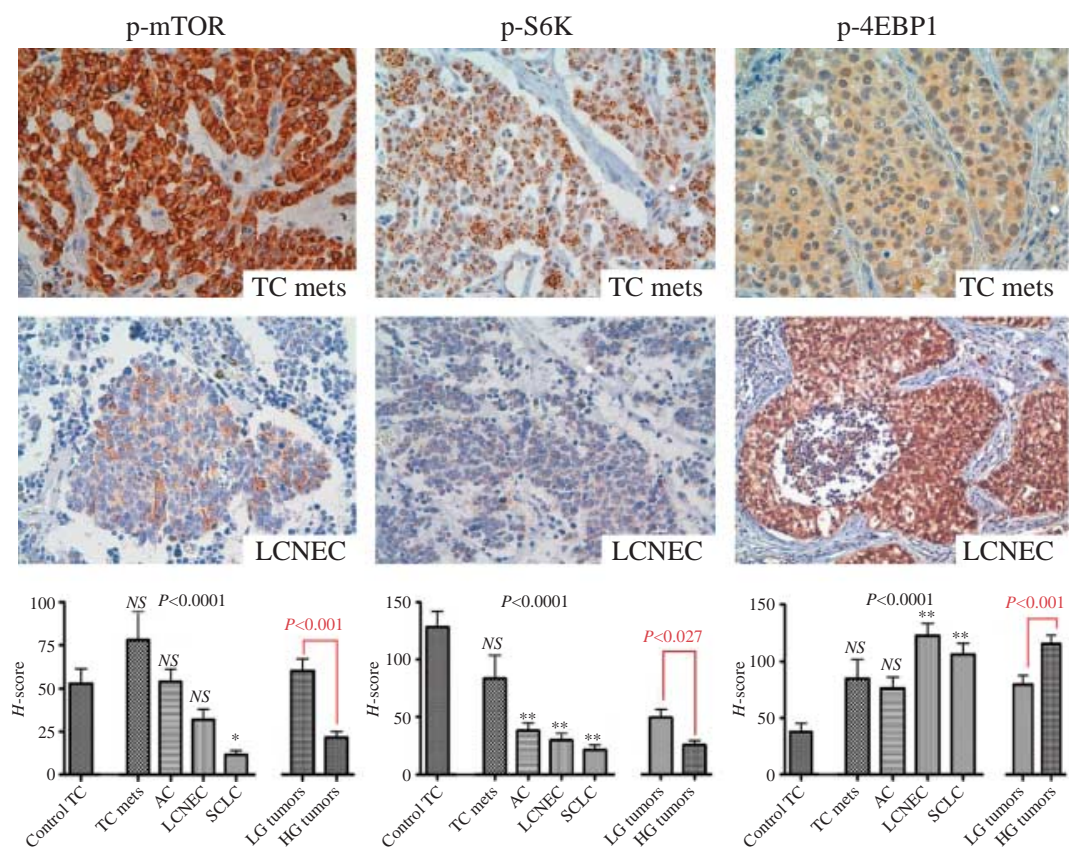

Figure 1 Immunohistochemical distribution of p-mTOR, p-S6K, and p-4EBP1 in lung NETs. Upper panels illustrate an intense p-mTOR and p-S6K as well as a weak p-4EBP1 expression in a case of TC mets as compared with a case of LCNEC (middle panels) showing the opposite features (immunoperoxidase, 200-fold magnification). In the lower panels, the histograms of mTOR-related molecules distribution are represented (with levels of significance for overall differences in black, for LG as compared with HG tumors in red, and for individual groups as compared with control TC at the top of each column. NS, not significant; ${ }^{*} P<0.01 ;{ }^{\star \star} P<0.001$ ). TC mets, typical carcinoids with lymph node metastases; AC, atypical carcinoids; LCNEC, large cell neuroendocrine carcinoma; SCLC, small cell lung carcinoma; LG tumors, low-grade tumors, including TCs with metastases and ACs; HG tumors, high-grade carcinomas, including LCNEC and small cell neuroendocrine carcinomas.

SCLC cases. This may possibly reflect unspecific binding of the antibody with unphosphorylated 4EBP1 in immunohistochemical sections (Fig. 2).

\section{Clinical pathological associations}

The distribution of p-mTOR, p-S6K, and p-4EBP1 expressions according to $H$-score values and clinical pathological variables is shown in Table 3.

P-mTOR and its downstream effectors did not correlate with proliferation or disease stage. By contrast, in LG tumors, high p-mTOR expression

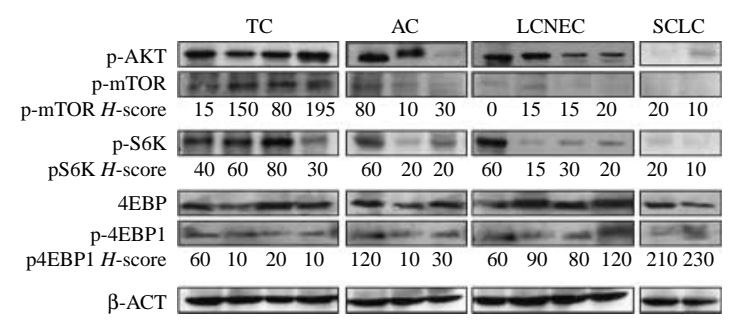

Figure 2 Western blot analysis of 13 cases of lung NETs. Immunohistochemical scores for p-mTOR, p-S6K, and $\mathrm{p}-4 \mathrm{EBP} 1$ in these same cases are reported for comparison. TC, typical carcinoid; AC, atypical carcinoid; LCNEC, large cell neuroendocrine carcinoma; SCLC, small cell lung carcinoma. associated with the parameters is indicative of a more favorable outcome, such as negative nodal status (in AC group, $P=0.016)$ and disease-free status $(P=0.005)$. P-S6K followed the same association, although slightly below statistical significance, whereas p-4EBP1 did not. Moreover, p-S6K and p-4EBP1 were expressed at higher levels in small tumors, with a strong significance in $\mathrm{LG}(\mathrm{p}-\mathrm{S} 6 \mathrm{~K}, P=0.006)$ and $\mathrm{HG}(\mathrm{p}-4 \mathrm{EBP} 1$, $P=0.008)$ tumors respectively.

No association was found among p-mTOR, p-S6K, and $\mathrm{p}-4 \mathrm{EBP} 1$ and overall survival, in either LG or HG tumors.

\section{Correlation among mTOR signaling and SSTR expression}

The functional activation of mTOR signaling pathway was defined by analyzing the correlation of expression between p-mTOR and its downstream molecules. A strong positive correlation was observed between p-mTOR and its effector p-S6K. The strong correlation was maintained in LG and HG tumors when analyzed separately. P-4EBP1 weakly correlated with p-mTOR but maintained a significant association with p-S6K (Table 4). 
Moreover, data obtained by our group (Righi et al. 2010) on the distribution of SSTR types $2 \mathrm{~A}$ and 3 in the same series (summarized in Table 1), were compared with the present findings, and a positive correlation was detected between p-mTOR (and p-S6K) and SSTR type 2A. This finding was evident both in LG and HG tumor groups $(P=0.034$ and $P=0.0075$ respectively). In addition, though to a lower extent, $\mathrm{p}$-mTOR correlated with SSTR type 3 expression $(P=0.034)$. By contrast, p-4EBP1 did not correlate with SSTR's expression.

Table 3 Distribution of the phosphorylated mammalian target of rapamycin (p-mTOR), p-S6K and p-4EBP1 expression levels according to clinical and pathological variables in 218 aggressive pulmonary neuroendocrine tumors

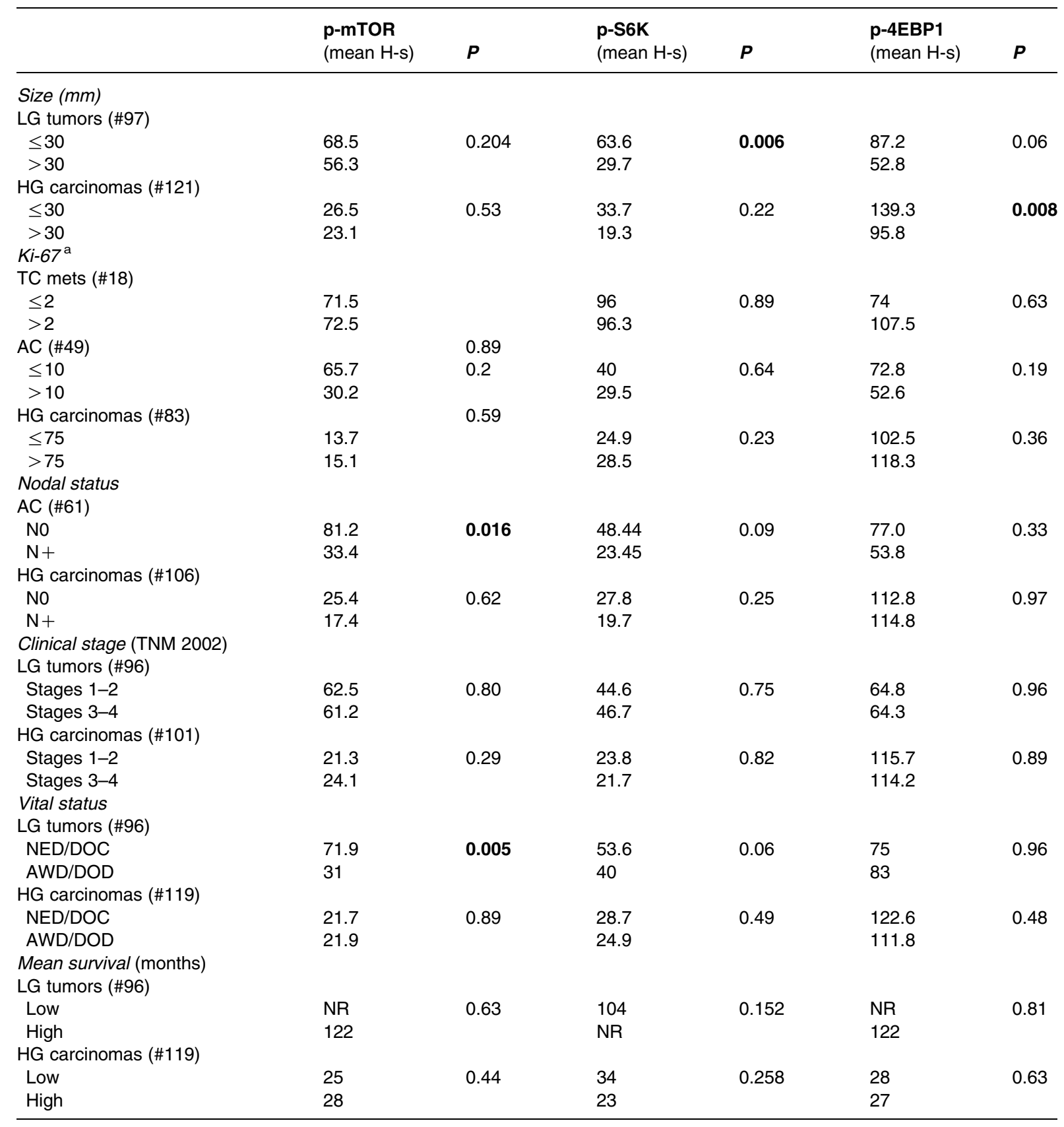

$\mathrm{H}$-s, $\mathrm{H}$-score; TC mets, typical carcinoids with metastases; AC, atypical carcinoids; LG tumors, low-grade tumors, including typical carcinoids with metastases and atypical carcinoids; HG carcinomas, high-grade carcinomas, including large and small cell neuroendocrine carcinomas; NED, not evidence of disease; DOC, death for other causes; AWD, alive with disease; DOD, dead of disease; NR, not reached. Bold denotes statistically significant values.

${ }^{a}$ Cut off values of $\mathrm{Ki}-67$ correspond to medians in each group. 
Table 4 Correlation of phosphorylated mammalian target of rapamycin (p-mTOR), p-S6K and 4EBP1 with somatostatin receptors in 218 pulmonary neuroendocrine tumors

\begin{tabular}{|c|c|c|c|}
\hline & p-mTOR & p-S6K & p-4EBP1 \\
\hline p-mTOR & - & $\begin{array}{l}P<0.0001 \\
r=0.458\end{array}$ & $\begin{array}{l}P=0.0074 \\
r=0.181\end{array}$ \\
\hline $\mathrm{p}-\mathrm{S} 6 \mathrm{~K}$ & - & - & $\begin{array}{l}P<0.0001 \\
r=0.312\end{array}$ \\
\hline SSTR-2A & $\begin{array}{l}P<0.0001 \\
r=0.271\end{array}$ & $\begin{array}{l}P=0.046 \\
r=0.135\end{array}$ & $\begin{array}{l}P=0.7 \\
r=-0.023\end{array}$ \\
\hline SSTR-3 & $\begin{array}{l}P=0.034 \\
r=0.271\end{array}$ & $\begin{array}{l}P=0.9 \\
r=-0.069\end{array}$ & $\begin{array}{l}P=0.9 \\
r=0.016\end{array}$ \\
\hline
\end{tabular}

SSTR, somatostatin receptor. Bold denotes statistically significant values.

\section{Differential inhibition of mTOR pathway in lung carcinoid cell lines}

H720 and H727 carcinoid cell lines showed a differential sensitivity to mTOR inhibition. H727 cell viability was not significantly modified by either rapamycin or RAD001 treatments, whereas H720 cells were sensitive to both the treatments with a dose-dependent trend (Fig. 3). Western blot analysis showed that in both the cell lines rapamycin and RAD001 differentially modified the phosphorylation status of the targets. Rapamycin in both the models mainly decreased 4EBP1 phosphorylation without a significant influence on p-mTOR, p-AKT, and p-S6K status. By contrast, RAD001 showed a differential modulation of p-AKT and p-S6K in sensitive $\mathrm{H} 720$ and insensitive $\mathrm{H} 727$ cell lines, with an unexpected increase in AKT associated with a decreased S6K phosphorylation in $\mathrm{H} 727$ and an inverse pattern in H720 cells. P-mTOR was higher in insensitive H727 cells, but was not significantly modulated by mTOR inhibition in both cell line models.

\section{Discussion}

In this study, we present a wide mapping of activated mTOR signaling pathway in pulmonary NETs, with a specific focus on aggressive forms of these tumors, which are a challenge for the correct clinical management and could benefit from mTOR-targeted therapies.

The functional activation of the PI3K/AKT/mTOR signaling pathway has never been extensively investigated in the spectrum pulmonary or other NETs, except for indirect evidence of the expression of functionally related molecules such as PTEN (Wang et al. 2002), tuberous sclerosis complex (TSC; Yao 2007), AKT (Shah et al. 2006), and IGF1R (von Wichert et al. 2000, Missiaglia et al. 2010).
Nevertheless, the clinical interest in mTOR has increased in recent years, since the development of selective inhibitors, and several preclinical trials have been conducted to test their efficacy in different human malignancies, including NETs (Duran et al. 2006, Kulke 2007, Zitzmann et al. 2007). With special reference to bronchial carcinoids, very recently the anti-proliferative effect of mTOR inhibitors in lung carcinoid primary cultures has been demonstrated in association with mTOR signaling down-modulation, and high mTOR mRNA and protein levels have been correlated with in vitro response (Zatelli et al. 2010). However, controversial results in terms of clinical response to mTOR inhibitors have been obtained in NET patients, possibly reflecting the heterogeneity of mTOR pathway functional status among different NET entities and within individual tumors of the same histotype.

A growing body of the literature regarding molecular drugs, such as EGFR tyrosine kinase inhibitors, supports the view that the appropriate selection of patients is always crucial to optimize such therapy benefits. Therefore, the clinical effort on the development of mTOR targeting therapies should be guided by the definition of its pathway activation status within individual NETs, also identifying specific profiles of pathogenetic and predictive interest. In clinical trials with mTOR inhibitors so far conducted in NETs (Yao et al. 2008, 2010), no tissue localization of mTOR or other molecules was determined as a potential predictor or response, whereas the few studies dealing with tissue distribution of mTOR and its related molecules in NETs (i.e. in the gastroenteropancreatic system) (Shida et al. 2010) lack the information of clinical response of patients to mTOR inhibitors. In this context, immunohistochemistry is the most reliable, reproducible, cost-effective, and

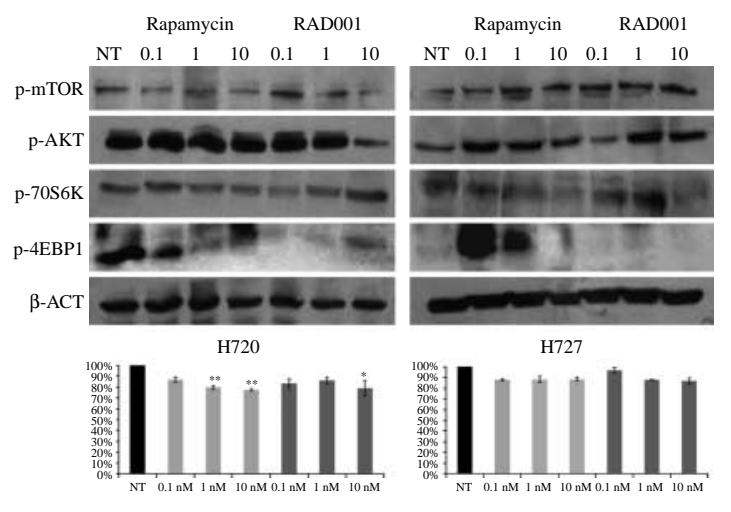

Figure 3 Western blot analysis and MTT assay of $\mathrm{H} 720$ and $\mathrm{H} 727$ lung carcinoid cell lines. ${ }^{*} P<0.05$ as compared with untreated cells (NT); ${ }^{\star \star} P<0.001$ as compared with NT. 
clinically applicable technique to investigate large tumor series, after assuming that specific antibodies against phosphorylated (active) forms of the target molecules are used through a semi-quantitative evaluation. An example supporting this point of view derives from a phase II trial about the effect of temsirolimus (a rapamycin derivative) in advanced NE carcinomas. Although this study concluded that this agent had little activity, not warranting further singleagent evaluation in this neoplastic setting, it was clearly shown that temsirolimus inhibited S6K phosphorylation and that higher baseline levels and lower levels after the therapy of p-mTOR were predictive factors of a better response (Duran et al. 2006).

Our study demonstrates that mTOR is consistently found in pulmonary NETs of different histological types, in correlation with its downstream molecules as confirmed also by western blot analysis, with a higher expression in low-to-intermediate-grade tumors. Since mTOR is associated with the control of cell proliferation, the lower activation in HG tumors is partially unexpected, although no data on HG NE carcinomas, pulmonary or extra-pulmonary, were available. As a matter of fact, all clinical trials with mTOR inhibitors conducted so far are designed onto advanced welldifferentiated/LG NE neoplasms. The present findings suggest that, at least in lung NETs, mTOR inhibition therapeutic strategy might be more effective in LG NE carcinomas. The correlation of mTOR was stronger and more significant with $\mathrm{p}$-S6K that is directly involved in mTOR signaling cascade (Guertin \& Sabatini 2007) than with p-4EBP1 that, conversely, may be phosphorylated by other kinases too (Heesom et al. 2001, Wang et al. 2003). As an alternative explanation for this discrepancy, p-4EBP1 determination by means of immunohistochemistry might be affected in some cases by the unspecific staining of unphosphorylated 4EBP1 protein, as detected in some cases of our series where immunohistochemistry and western blot tests were compared. Moreover, the heterogeneous distribution of the molecules under investigation within individual histological subtypes was paralleled by in vitro experiments on two carcinoid cell lines that showed a differential response to rapamycin and RAD001 treatments, with heterogeneous modulation of mTOR-related molecules, potentially supporting that different activation status might be responsible for different responses. These features support the existence of different functional levels of the pathway, and reinforces the contention that typing different mTOR-pathway-related molecules might help to correctly select patients for mTOR-inhibitor-guided treatments (Meric-Bernstam
\& Gonzalez-Angulo 2009). In this respect, a weakness of this study is its retrospective character and the lack of clinical correlates between mTOR and related molecule(s) expression, and the clinical response of patients to mTOR-inhibitor treatments. This limitation partly reflects the current lack of standardized therapeutic approaches to the use of these drugs in the setting of clinically aggressive lung NETs.

Another interesting finding is the correlation that mTOR demonstrated with SSTR expression of both types $2 \mathrm{~A}$ and 3 . Biologically, this observation seems to support the view that mTOR activity might be modulated also by SSTR in the light of experimental observations on octreotide capability to down-regulate mTOR-upstream molecules, such as PI3K and AKT, eventually leading to anti-proliferative activity (Theodoropoulou et al. 2006). Moreover, SSTR may indirectly modulate mTOR pathway activation through the interaction with IGF1 axis, since octreotide has been demonstrated to decrease IGF1 serum levels and repress its gene transcription (Ferjoux et al. 2000). Such cross talk between SSTRs and mTOR might explain the results of recent in vitro and in vivo studies on the anti-tumoral efficacy of combined mTOR inhibitor and octreotide treatment in NETs (Grozinsky-Glasberg et al. 2008, Moreno et al. 2008, Yao et al. 2008).

In the current tumor series, all the mTOR-related molecules failed to show a significant impact on overall survival. However, higher levels of p-mTOR and $\mathrm{p}$-S6K expression were associated with more favorable clinico-pathological parameters, for example, they were found in the LG tumor groups and associated with negative nodal status (in the AC group only) or with the disease-free status (in the LG tumor group). By contrast, 4EBP1 was unrelated to clinical pathological characteristics, suggesting activation from alternative kinase pathways other than mTOR. The literature on the prognostic role of mTOR pathway activation status in human tumors is scanty and controversial, supporting a favorable impact on some models, such as ovarian cancer (Noske et al. 2008), and an adverse prognostic effect on others, such as renal cell (Pantuck et al. 2007, Campbell et al. 2008), breast (Noh et al. 2008) and biliary tract (Herberger et al. 2007) carcinomas. A single study (Missiaglia et al. 2010) on pancreatic endocrine tumors indirectly associated the up modulation of mTOR pathway (via the deregulation of TSC2 and PTEN proteins) with aggressive disease, but the most significant association was found with malignant phenotype and loss of differentiation, and neither TSC2 nor PTEN proteins were associated with 
prognosis at multivariate analysis. Conversely, in our series of lung NETs, mTOR expression was higher in LG tumors as compared with HG poorly differentiated types, and in specific subgroups was associated with favorable parameters such as negative nodal status and free-of-disease status. Such findings might reflect tumor-specific activation status of mTOR pathway and are possibly related to specific properties on cellular growth rather than proliferation control in our tumor model. In conclusion, we described the activation pattern of mTOR/S6K/4EBP1 signaling pathway in a large series of aggressive pulmonary NETs, also providing evidence for cross talking with the SSTR pathway. These data support the concept that a detailed protein mapping of mTOR-pathway-related molecules in lung (and possibly other) NETs may drive a more selective strategy for targeting mTOR in individual NETs.

\section{Declaration of interest}

The authors declare that there is no conflict of interest that could be perceived as prejudicing the impartiality of the research reported.

\section{Funding}

This work is supported by the grants from the Italian Ministry of University (ex 60\% to M Volante and M Papotti) and from the un-restricted research grants from Novartis Pharma to $M$ Volante (FT75/08) and M Papotti (FT81/07).

\section{Acknowledgements}

We are grateful to Prof. G Bussolati and F Ardissone (University of Turin) and Prof. G Rindi and M Rusca (University of Parma) for their helpful suggestions.

\section{References}

Bjornsti MA \& Houghton PJ 2004 The TOR pathway: a target for cancer therapy. Nature Reviews. Cancer 4 335-348. (doi:10.1038/nrc1362)

Campbell L, Jasani B, Edwards K, Gumbleton M \& Griffiths DF 2008 Combined expression of caveolin-1 and an activated AKT/mTOR pathway predicts reduced disease-free survival in clinically confined renal cell carcinoma. British Journal of Cancer 98 931-940. (doi:10.1038/sj.bjc.6604243)

Cappia S, Righi L, Mirabelli D, Ceppi P, Bacillo E, Ardissone F, Molinaro L, Scagliotti GV \& Papotti M 2008 Prognostic role of osteopontin expression in malignant pleural mesothelioma. American Journal of Clinical Pathology 130 58-64. (doi:10.1309/TWCQV536W WRNEU51)
Dancey JE 2006 Therapeutic targets: mTOR and related pathways. Cancer Biology \& Therapy 5 1065-1073.

Duran I, Kortmansky J, Singh D, Hirte H, Kocha W, Goss G, Le L, Oza A, Nicklee T, Ho J et al. 2006 A phase II clinical and pharmacodynamic study of temsirolimus in advanced neuroendocrine carcinomas. British Journal of Cancer 95 1148-1154. (doi:10.1038/sj.bjc.6603419)

Duran I, Salazar R, Casanovas O, Arrazubi V, Vilar E, Siu LL, Yao J \& Tabernero J 2007 New drug development in digestive neuroendocrine tumors. Annals of Oncology 18 1307-1313. (doi:10.1093/annonc/mdm009)

Ferjoux G, Bousquet C, Cordelier P, Benali N, Lopez F, Rochaix P, Buscail L \& Susini C 2000 Signal transduction of somatostatin receptors negatively controlling cell proliferation. Journal of Physiology 94 205-210. (doi:10. 1016/S0928-4257(00)00206-0)

Garcia-Yuste M, Matilla JM \& Gonzalez-Aragoneses F 2008 Neuroendocrine tumors of the lung. Current Opinion in Oncology 20 148-154. (doi:10.1097/CCO. 0b013e3282f35ed3)

Grozinsky-Glasberg S, Franchi G, Teng M, Leontiou CA, Ribeiro de Oliveira A Jr, Dalino P, Salahuddin N,

Korbonits M \& Grossman AB 2008 Octreotide and the mTOR inhibitor RAD001 (everolimus) block proliferation and interact with the Akt-mTOR-p70S6K pathway in a neuro-endocrine tumour cell line. Neuroendocrinology 87 168-181. (doi:10.1159/ 000111501)

Grozinsky-Glasberg S, Rubinfeld H, Nordenberg Y, Gorshtein A, Praiss M, Kendler E, Feinmesser R, Grossman AB \& Shimon I 2010 The rapamycinderivative RAD001 (everolimus) inhibits cell viability and interacts with the Akt-mTOR-p70S6K pathway in human medullary thyroid carcinoma cells. Molecular and Cellular Endocrinology 315 87-94. (doi:10.1016/ j.mce.2009.09.027)

Guertin DA \& Sabatini DM 2007 Defining the role of mTOR in cancer. Cancer Cell 12 9-22. (doi:10.1016/j.ccr.2007. 05.008)

Heesom KJ, Gampel A, Mellor H \& Denton RM 2001 Cell cycle-dependent phosphorylation of the translational repressor eIF-4E binding protein-1 (4E-BP1). Current Biology 11 1374-1379. (doi:10.1016/S09609822(01)00422-5)

Herberger B, Puhalla H, Lehnert M, Wrba F, Novak S, Brandstetter A, Gruenberger B, Gruenberger T, Pirker R \& Filipits M 2007 Activated mammalian target of rapamycin is an adverse prognostic factor in patients with biliary tract adenocarcinoma. Clinical Cancer Research 13 4795-4799. (doi:10.1158/1078-0432.CCR-07-0738)

Huang WC, Xie Z, Konaka H, Sodek J, Zhau HE \& Chung LW 2005 Human osteocalcin and bone sialoprotein mediating osteomimicry of prostate cancer cells: role of cAMP-dependent protein kinase A signaling pathway. Cancer Research 65 2303-2313. (doi:10.1158/00085472.CAN-04-3448) 
Kulke MH 2007 New developments in the treatment of gastrointestinal neuroendocrine tumors. Current Oncology Reports 9 177-183. (doi:10.1007/s11912-007-0019-y)

Lam JS, Pantuck AJ, Belldegrun AS \& Figlin RA 2007 Protein expression profiles in renal cell carcinoma: staging, prognosis, and patient selection for clinical trials. Clinical Cancer Research 13 703s-708s. (doi:10.1158/ 1078-0432.CCR-06-1864)

Meric-Bernstam F \& Gonzalez-Angulo AM 2009 Targeting the mTOR signaling network for cancer therapy. Journal of Clinical Oncology 27 2278-2287. (doi:10. 1200/JCO.2008.20.0766)

Missiaglia E, Dalai I, Barbi S, Beghelli S, Falconi M, della Peruta M, Piemonti L, Capurso G, Di Florio A, delle Fave G et al. 2010 Pancreatic endocrine tumors: expression profiling evidences a role for AKT-mTOR pathway. Journal of Clinical Oncology 28 245-255. (doi:10.1200/JCO.2008.21.5988)

Mita MM, Mita AC, Chu QS, Rowinsky EK, Fetterly GJ, Goldston M, Patnaik A, Mathews L, Ricart AD, Mays T et al. 2008 Phase I trial of the novel mammalian target of rapamycin inhibitor deforolimus (AP23573; MK-8669) administered intravenously daily for 5 days every 2 weeks to patients with advanced malignancies. Journal of Clinical Oncology 26 361-367. (doi:10.1200/JCO.2007. 12.0345)

Moreno A, Akcakanat A, Munsell MF, Soni A, Yao JC \& Meric-Bernstam F 2008 Antitumor activity of rapamycin and octreotide as single agents or in combination in neuroendocrine tumors. Endocrine-Related Cancer 15 257-266. (doi:10.1677/ERC-07-0202)

Noh WC, Kim YH, Kim MS, Koh JS, Kim HA, Moon NM \& Paik NS 2008 Activation of the mTOR signaling pathway in breast cancer and its correlation with the clinicopathologic variables. Breast Cancer Research and Treatment 110 477-483. (doi:10.1007/s10549-0079746-x)

Noske A, Lindenberg JL, Darb-Esfahani S, Weichert W, Buckendahl AC, Roske A, Sehouli J, Dietel M \& Denkert C 2008 Activation of mTOR in a subgroup of ovarian carcinomas: correlation with p-eIF-4E and prognosis. Oncology Reports 20 1409-1417. (doi:10. 3892/or_00000160)

Pandya KJ, Dahlberg S, Hidalgo M, Cohen RB, Lee MW, Schiller JH \& Johnson DH 2007 A randomized, phase II trial of two dose levels of temsirolimus (CCI-779) in patients with extensive-stage small-cell lung cancer who have responding or stable disease after induction chemotherapy: a trial of the Eastern Cooperative Oncology Group (E1500). Journal of Thoracic Oncology 2 1036-1041. (doi:10.1097/JTO.0b013e318155a439)

Pantuck AJ, Seligson DB, Klatte T, Yu H, Leppert JT, Moore L, O'Toole T, Gibbons J, Belldegrun AS \& Figlin RA 2007 Prognostic relevance of the mTOR pathway in renal cell carcinoma: implications for molecular patient selection for targeted therapy. Cancer 109 2257-2267. (doi:10.1002/cncr.22677)
Pelosi G, Volante M, Papotti M, Sonzogni A, Masullo M \& Viale G 2006 Peptide receptors in neuroendocrine tumors of the lung as potential tools for radionuclide diagnosis and therapy. Quarterly Journal of Nuclear Medicine and Molecular Imaging 50 272-287.

Podsypanina K, Lee RT, Politis C, Hennessy I, Crane A, Puc J, Neshat M, Wang H, Yang L, Gibbons J et al. 2001 An inhibitor of mTOR reduces neoplasia and normalizes p70/S6 kinase activity in Pten $+/-$ mice. PNAS 98 10320-10325. (doi:10.1073/pnas.171060098)

Righi L, Volante M, Tavaglione V, Billè A, Daniele L, Angusti T, Inzani F, Pelosi G, Rindi G \& Papotti M 2010 Somatostatin receptor tissue distribution in lung neuroendocrine tumours: a clinicopathologic and immunohistochemical study of 218 "clinically aggressive” cases. Annals of Oncology 21 548-555. (doi:10.1093/annonc/mdp334)

Shah T, Hochhauser D, Frow R, Quaglia A, Dhillon AP \& Caplin ME 2006 Epidermal growth factor receptor expression and activation in neuroendocrine tumours. Journal of Neuroendocrinology 18 355-360. (doi:10. 1111/j.1365-2826.2006.01425.x)

Shida T, Kishimoto T, Furuya M, Nikaido T, Koda K, Takano S, Kimura F, Shimizu H, Yoshidome H, Ohtsuka M et al. 2010 Expression of an activated mammalian target of rapamycin (mTOR) in gastroenteropancreatic neuroendocrine tumors. Cancer Chemotherapy and Pharmacology 65 889-893. (doi:10.1007/s00280-0091094-6)

Srirajaskanthan R, Toumpanakis C, Karpathakis A, Marelli L, Quigley AM, Dusmet M, Meyer T \& Caplin ME 2009 Surgical management and palliative treatment in bronchial neuroendocrine tumours: a clinical study of 45 patients. Lung Cancer 65 68-73. (doi:10.1016/ j.lungcan.2008.10.025)

Theodoropoulou M, Zhang J, Laupheimer S, Paez-Pereda M, Erneux C, Florio T, Pagotto U \& Stalla GK 2006 Octreotide, a somatostatin analogue, mediates its antiproliferative action in pituitary tumor cells by altering phosphatidylinositol 3-kinase signaling and inducing Zac1 expression. Cancer Research 66 1576-1582. (doi:10.1158/0008-5472.CAN-05-1189)

Travis WD, Brambilla E, Muller-Hermelink HK \& Harris CC 2004 Tumours of the Lung, Pleura, Thymus and Heart. Lyon: IARC Press.

Vignot S, Faivre S, Aguirre D \& Raymond E 2005 mTORtargeted therapy of cancer with rapamycin derivatives. Annals of Oncology 16 525-537. (doi:10.1093/annonc/ mdi113)

Wang L, Ignat A \& Axiotis CA 2002 Differential expression of the PTEN tumor suppressor protein in fetal and adult neuroendocrine tissues and tumors: progressive loss of PTEN expression in poorly differentiated neuroendocrine neoplasms. Applied Immunohistochemistry \& Molecular Morphology 10 139-146. (doi:10.1097/00022744200206000-00008) 
Wang X, Li W, Parra JL, Beugnet A \& Proud CG 2003 The $\mathrm{C}$ terminus of initiation factor $4 \mathrm{E}$-binding protein 1 contains multiple regulatory features that influence its function and phosphorylation. Molecular and Cellular Biology 23 1546-1557. (doi:10.1128/MCB.23.5.15461557.2003)

von Wichert G, Jehle PM, Hoeflich A, Koschnick S, Dralle H, Wolf E, Wiedenmann B, Boehm BO, Adler G \& Seufferlein T 2000 Insulin-like growth factor-I is an autocrine regulator of chromogranin A secretion and growth in human neuroendocrine tumor cells. Cancer Research 60 4573-4581.

Yao JC 2007 Neuroendocrine tumors. Molecular targeted therapy for carcinoid and islet-cell carcinoma. Best Practice \& Research. Clinical Endocrinology \& Metabolism 21 163-172. (doi:10.1016/j.beem. 2007.01.006)

Yao JC, Phan AT, Chang DZ, Wolff RA, Hess K, Gupta S, Jacobs C, Mares JE, Landgraf AN, Rashid A et al. 2008 Efficacy of RAD001 (everolimus) and octreotide LAR in advanced low- to intermediate-grade neuroendocrine tumors: results of a phase II study. Journal of Clinical Oncology 26 4311-4318. (doi:10.1200/JCO.2008.16.7858)

Yao JC, Lombard-Bohas C, Baudin E, Kvols LK, Rougier P, Ruszniewski P, Hoosen S, St Peter J, Haas T, Lebwohl D et al. 2010 Daily oral everolimus activity in patients with metastatic pancreatic neuroendocrine tumors after failure of cytotoxic chemotherapy: a phase II trial. Journal of Clinical Oncology 28 69-76. (doi:10.1200/ JCO.2009.24.2669)

Zatelli MC, Minoia M, Martini C, Tagliati F, Ambrosio MR, Schiavon M, Buratto M, Calabrese F, Gentilin E, Cavallesco G et al. 2010 Everolimus as a new potential antiproliferative agent in aggressive human bronchial carcinoids. Endocrine-Related Cancer 17 719-729. (doi:10.1677/ERC-10-0097)

Zitzmann K, De Toni EN, Brand S, Goke B, Meinecke J, Spottl G, Meyer HH \& Auernhammer CJ 2007 The novel mTOR inhibitor RAD001 (everolimus) induces antiproliferative effects in human pancreatic neuroendocrine tumor cells. Neuroendocrinology 85 54-60. (doi:10.1159/ 000100057) 